\title{
Creation of a computational framework for the European transmission grid with Power-to-Gas
}

\author{
Andrea Mazza, Andrea Rogin, Shaghayegh Zalzar, Abouzar Estebsari, Ettore Bompard \\ Dipartimento Energia "Galileo Ferraris" \\ Politecnico di Torino \\ Torino, Italy \\ \{andrea.mazza, shaghayegh.zalzar, abouzar.estebsari, ettore.bompard\}@polito.it
}

\begin{abstract}
The presence of high penetration of Variable Renewable Energy Sources (VRES) is one of the key aspects of the modern electricity system. The new challenges to be faced require novel technologies which enhance the flexibility of the transmission system. In this paper, the exploitation of the power-to-gas technology (PtG) is considered as a solution for the flexibility challenges, allowing to absorb the excess of electricity produced by VRES and at the same time, producing synthetic natural gas (SNG). This work presents a computational framework based on DC Optimal Power Flow capable to simulate the day-ahead market and the following intra-day market, applied to a simplified European transmission network, by considering different scenarios for both load and generation. PtG plants model are modelled based on the real measurements of a 2-MW Alkaline (AEC) electrolyser. The results of a given PtG placement configuration, applied to a current scenario (2017) and two future scenarios $(2030 \& 2040)$ show that the fast response of PtG units improves the system performance and reduces the VRESs' imbalance up to $\sim \mathbf{9 0} \%$ in terms of imbalance time duration and $\sim \mathbf{4 0} \%$ in terms of peak imbalance power. These results lead to further investigations, about the optimal PtG units' placement and sizing, as well as their economic and technical consequences on the transmission network operation.
\end{abstract}

Keywords-Transmission system, power-to-gas, VRES, electricity market, European transmission grid flexibility.

\section{INTRODUCTION}

The recent trend toward increasing the penetration of Variable Renewable Energy Sources (VRES) in power systems has arisen new challenges for the system operation. The integration of VRESs into the power systems has changed the paradigm of the system control and network management both in distribution and transmission level, due to the unpredictability and uncontrollability of these sources.

The studies involving the distribution system and VRES focus on how to pass from a passive to an active network management by handling with the issues arising by high penetration of VRES, such as reverse power flow [1], implementation of new voltage control strategies [2], managing loading conditions [3] as well as the use of the VRES for creating an independent system (by exploiting the concept of microgrid [4]) or improving the performance of the network through the application of optimization methods (e.g., probability-based ones [5]).

The recent studies focusing on transmission system tried to figure out how to reach up to $100 \%$ Renewable Energy Sources (RES) scenarios [6], modeling the integration of VRES (non controllable) and "traditional" RES (like hydro, which is controllable) in generation expansion planning [7], and managing the transmission network expansion taking into account the uncertainties introduced by VRES [8][9]. Furthermore, new computational tools have been proposed to investigate the complexity of the high-VRES scenarios in power systems and the appropriate solutions [10][11].

One of the key aspects for reaching 100\% RES penetration in power systems is the exploitation of different storage technologies, which can act as buffers against the uncertainty and variability of the ever-increasing VRESs [12].

The term "storage" can be associated both to devices characterized by conversion and storage of electricity (i.e., batteries) and to devices which allow to make the conversion of the electricity in another energy carrier. In the latter case, the carrier can be stored in a dedicated tank, or infeed into an existent infrastructure, enabling the creation of a multi-carrier system. In this paper we focus on the application of a conversion system called Power-to-Gas (PtG) into the transmission system operation. This technology has recently gained increasing interests because of the possibility to handle with the long-term storage [13].

The term PtG implies on the production of both hydrogen or synthetic natural gas (SNG) (as shown in Fig. 1), and in particular this paper focuses on the use of the complete chain (i.e., the power-to-SNG process) [14].

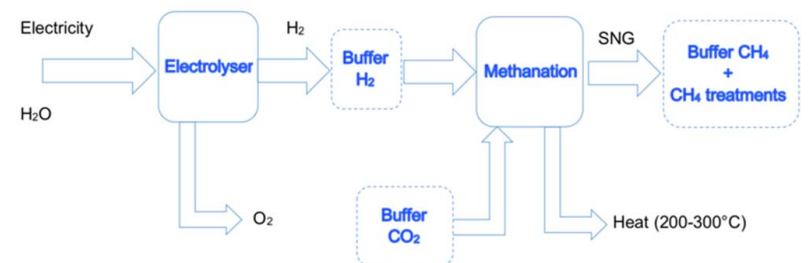

Fig. 1. Diagram of the complete chain for power-to-SNG Scenario

The study of the deployment of any new technology into the electricity network needs both the proper modelling of that technology and the implementation of the model on a meaningful network. In this regard, this paper aims to present a computational framework representing the European power system operation and the effect of PtG integration on the transmission system operation through a market-based approach. The transmission system operation has been simulated with hourly load and VRES production in dayahead market model. Then, intraday market is modelled taking into account 10-minute variations of VRES production, leading to a rescheduling of the conventional generators to maintain supply-demand balance. The presence of additional responsive loads (such as PtG plants) allows to reduce the need for rescheduling the conventional generators which in turn improves the efficiency of the generators and reduces the operation costs.

The paper is organized as follows: Section II presents the general idea for integrating the $\mathrm{PtG}$ plants into the transmission system. Section III focuses on the European 
network model, whereas Section IV aims to show where to get the data of loads and generation for feeding the network. Section V shows the results. Finally, the conclusions are provided in Section VI.

\section{THE EUROPEAN TRANSMISSION SYSTEM}

The detailed study of the integration of PtG into the transmission system needs the following features:

- Proper description of the network infrastructure: this means the implementation of a realistic transmission system, in terms of physical parameters (e.g., resistance, reactance, length, line thermal limits and so on).

- Geographical coverage: to analyse the impact of a new technology which aims to support the integration of RES in Europe, the geographical information are fundamental.

- Proper values of generation and loads: the generation mix and load data regarding the next decades (e.g., 2030 and 2040).

The model of the European transmission system related to the Ten Years Network Development Plan (TYNDP) 2016 [15] ("Stum Model") does not report any information regarding the geographical location of the different nodes, so could not be used in this study due to the lack of the second point of the feature list mentioned above.

On May 2018, an updated version of the model stored in the repository [16] was published which can be found at the web-link [17] and in the repository [18]. The model has been validated in [19]. The model simplifies the European network to an equivalent network model composed of 256 nodes, created by applying a k-means clustering technique at the European Network (over 6000 nodes) which was obtained from the European Network Map [20]. This simplified network model is fully composed of $380 \mathrm{kV}$ voltage level for transmission lines/transformers, connecting the different nodes. Due to the lack of information regarding the real lines composing the original network, a simplification has been carried out, i.e., defined lines geometries have been considered, as shown in TABLE I. [21].

TABLE I. TRANSMISSION LINES' PROPERTIES

\begin{tabular}{|c|c|c|}
\hline Voltage [kV] & Current limit [A] & Power limit [MVA] \\
\hline 220 & 1290 & 492 \\
\hline 300 & 1935 & 1005 \\
\hline 380 & 2580 & 1698 \\
\hline
\end{tabular}

The resulting network's summary is presented in TABLE II. , whereas the representation of the georeferenced model is shown in Fig. 2.

TABLE II. NETWORK \#T3 DATA SUMMARY

\begin{tabular}{|c|c|c|c|c|}
\hline Buses & Branches & DC lines & Generators & Load [GW] \\
\hline 257 & 460 & 24 & 828 & $\sim 360$ \\
\hline
\end{tabular}

The network covers all the ENTSOE countries and the load profiles in the original dataset, referred to the year 2013. The share of load for every cluster has been obtained by considering a combination between the population and Gross Domestic Product (GDP) of each cluster.
The generation in the original dataset also refers to the year 2013. According to this dataset, at every node of the network more than one type of generator is connected. The model also includes the DC links existing in Europe, considered as part of the model.

Both generation and loads have been updated according to current and future scenarios, as shown in the following Section III.

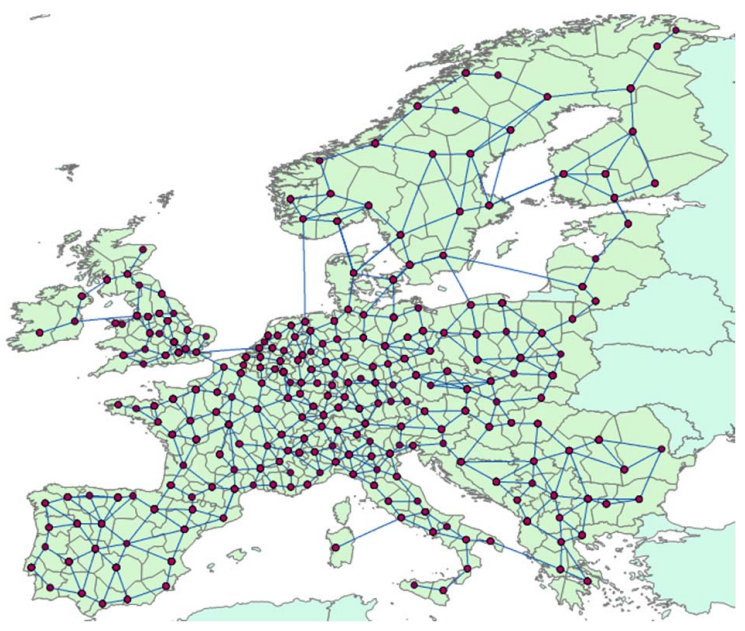

Fig. 2. Representation of the network \#T3

\section{DATA TO FEED THE NETWORK}

\section{A. Loads}

The values of loads and their variation with time have been updated on the basis of the 2017 data provided by [22], for the countries considered. The power statistics offer yearly historical data of power consumption, provided with a temporal resolution of one hour, and country level as spatial resolution. The country-level load has been distributed within the buses of the same country in proportion to the nominal load at each bus.

Beyond the most recent historical data, various scenarios can be used in the model. The selection of the scenario implies the choice of the desired load profile and scaled up baseline generation, based on the data obtained by [23]. The forecasts are part of the ENTSO-E TYNDP [15][24], which provides a detailed overview of possible European energy futures up to 2040.

In particular, the scripts are able to handle all the scenarios presented by ENTSO-E for 2030 and 2040 in [24], plus the current situation (referring to the year 2017).

\section{B. Generation}

Similar to the load profiles, generation capacity of each network has been updated to match 2017 data [25]. The number of generators and their network positions have been kept the same as in original network model, while the capacity of each generator type within each country has been scaled up according to the 2017 installed capacities. Minimum stable power output, and ramp rate values have been considered for each type of generator. The data has been collected from [26].

\section{MAIN CARACHTERISTICS OF THE SimUlation TOOL}

One of the main roles of $\mathrm{PtG}$ is to produce SNG by exploiting the excess of electricity produced by VRES, while 
the installation of a number of PtG plants can also help to stabilise the electricity network. In this framework, the PtG plants operate as balancing elements, through enabling longterm storage of the excess of electricity produced by VRES.

In this paper, the integration of PtG into the electricity system is modelled through their participation into the intraday market to solve the imbalances created by intraday VRES variations with respect to the day-ahead market results. The day-ahead market model consists on 1-hour time intervals while the intraday market includes 10-minute time intervals. Both markets are modelled by DC Optimal Power Flow (DCOPF) with the objective function of minimizing the total operation cost $f(x)$ of the electricity system in an optimization problem.

The two above mentioned OPF models aim to find the set of generators which lead to the supply of demand at minimum generation cost. In particular, the first OPF dispatches the expected value of VRES and the traditional generation through an economic merit order, whereas the second one aims to solve the imbalances caused by the variable nature of the VRES by re-dispatching the conventional generators and the flexibility provided by PtG units. These two OPF programs aim to represent the day-ahead market and a (quasi) real-time market, on which PtG can operate for providing its services to the network. The script developed in coding environment Matlab ${ }^{\circledR}$, and recalled the functions developed in Matpower [27].

\section{A. Day-ahead market}

As shown in Fig. 3, the Day-ahead Market (DAM) is modelled as a loop, in which each iteration represents an hour.

For each iteration:

- A function updates the time and all the time related variables, for example the nodal loads for the current hour, and the current PV and wind generation forecasts for the hour.

- Then a DCOPF is performed, and it provides the list of generators that are required online in order to supply optimally the load in that iteration, without violating any generator or branch constrain.

- This list of online generators is saved and assigned to the current hour.

- Since it is expected for the VRES production to have variations in the intra-day market, it is necessary to add more generators online in order to provide security/reserve /ramp services. These generators are chosen among the cheapest that could not participate to the day-ahead market, and the added capacity depends on two factors, seasonality and time.

- After adding additional generators, a DCOPF is performed, in order to obtain the market clearance for this iteration. A new iteration begins after the current output is saved.

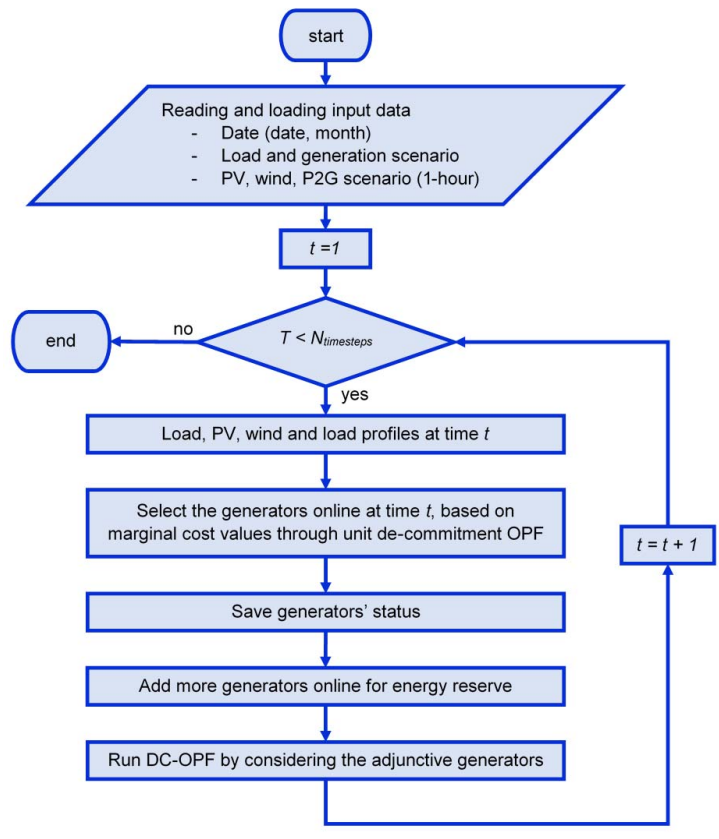

Fig. 3. DAM loop flochart.

When all the hours of the day have been processed, the day-ahead market algorithm ends.

Additional generators are necessary since there are two time-intervals in which the ramp service is highly required: the first is at sunrise when PV production rapidly increases, whereas the second one is at sunset, when PV production rapidly decreases. This is needed since in those hours the averages at five to fifteen minutes do not oscillate around the hourly averaged value, but steadily increase or decrease. Because of this, more generators are needed for ramp service. Seasonality changes the time when these two conditions occur, and this affects also the number of generators required to change along the year.

\section{B. Intraday-market}

The intraday market algorithm, as previously stated, shares most of the input part with the one emulating the dayahead market. Moreover, it receives as input the status of the generators for every hour from the day-ahead market. There is an hourly time loop that updates every variable that changes hourly (DA time loop), and, within each hour-iteration, another time loop represents the user defined time steps within the hour (ID time loop), e.g., twelve five-minutes time steps, six ten-minutes time steps, four fifteen-minutes time steps.

Within each ID time loop, the PV and wind profiles are averaged according to the user time-step, and the difference of the actual renewable power with respect to the hourly one is calculated for each PV/wind generator. Part of this difference between the actual ID generation and the DA forecast, can be assigned to every $\mathrm{PtG}$ unit as setpoint. If this quantity is positive it means that currently there is more $\mathrm{PV} /$ wind generation than forecasted, then PtG units can increase their power absorption. Vice versa, if this quantity is negative, there is less $\mathrm{PV} /$ wind generation than forecasted, therefore $\mathrm{PtG}$ units will have to lower their power absorption in order to help the network. 
When PtG units' set-points for the current ID time step are known, PtG model is launched for each PtG unit. The outputs of the model executions are the responses of PtG units in the current minute which equals as load values for dispatchable loads in Matpower modelling.

Since Matpower's OPF offers a static resolution of the network, the ramp constrains are enforced through the maximum and minimum power constrains of generators. In each iteration the generation results of the previous time iteration are taken in account, and the maximum/minimum power constrains are updated as the previous results plus/minus the ramp rate applied to the user-defined time step for ID market.

A DCOPF is performed, and the output is saved as the results for the current iteration, ending the iteration. The flowchart of real time market is shown in Fig. 4.

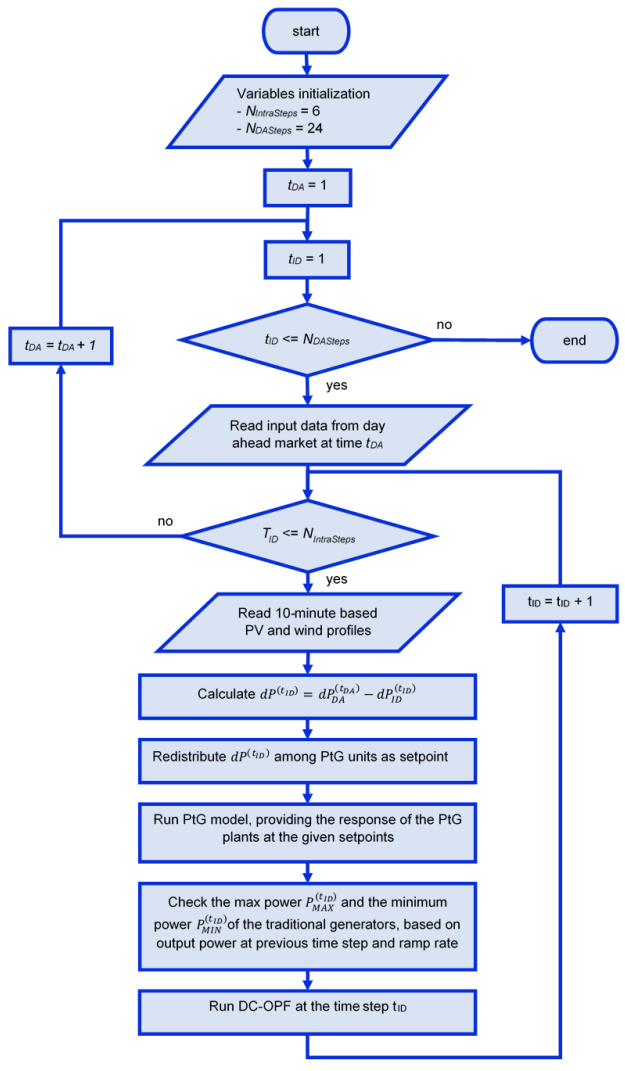

Fig. 4. Intraday market loop flochart.

\section{RESUlts}

\section{A. Current scenario: year 2017}

The results shown refer to two days of the year, in January and July, respectively.

Fig. 5 shows the total VRES variability from the forecasts of the day-ahead OPF to the actual values in intra-day OPF: this unbalance is representative of the whole network and has to be solved by involving the traditional generators, that should adapt their production for maintaining the system in operation. The same figure shows in dashed blue the effect of $10 \mathrm{GW}$ of PtG, working in different network nodes, on the imbalance of power of the whole network. The redistribution of the power among the different PtG has been based on size criterion. In this particular example, the sizes of the PtG plants has been fixed $1 \mathrm{GW}$.

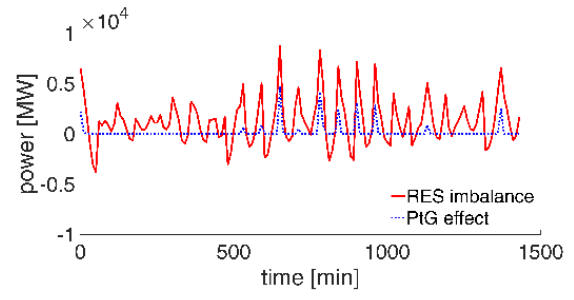

Fig. 5. VRES imbalance before and after the PtG installation (10th January, 2017 scenario)

The same results related to July are shown in Fig. 6: it is evident that there is more variability to be faced in presence of the sunrise and sunset, where the power produced by the sun is increasing/decreasing in monotonic way.

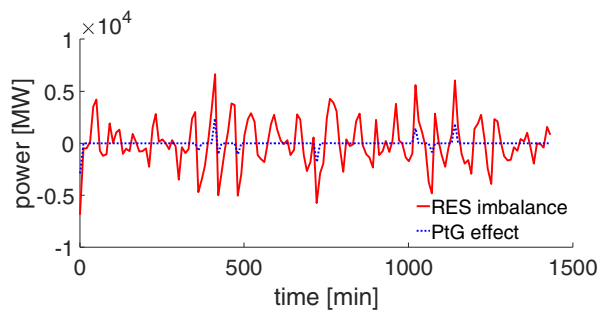

Fig. 6. RES imbalance before and after the PtG installation (3rd July, 2017 scenario)

The PtG effects for the two days of 2017 scenario are shown in TABLE III. : the presence of PtG, also in this case, limits both the duration and the peak of the imbalance, helping the operation of the transmission network.

TABLE III. PERFormance OF PTG IN 2017 SCENARIO

\begin{tabular}{|c|c|c|c|c|c|}
\hline \multirow{3}{*}{ Scenario } & \multirow{2}{*}{ PtG status } & \multicolumn{4}{|c|}{ VRES imbalance } \\
\cline { 3 - 4 } & & $\begin{array}{c}\text { Duration } \\
\text { [min] }\end{array}$ & $\begin{array}{c}\text { Difference } \\
\text { [\%] }\end{array}$ & $\begin{array}{c}\text { Peak } \\
\text { [MW] }\end{array}$ & $\begin{array}{c}\text { Difference } \\
\text { [\%] }\end{array}$ \\
\hline \multirow{3}{*}{ January } & Off & 1440 & \multirow{2}{*}{$-90 \%$} & 8758 & \multirow{2}{*}{\begin{tabular}{c} 
[ \\
\cline { 3 - 3 }
\end{tabular}} \\
\cline { 2 - 3 } July & On & 150 & & 4600 & $-47 \%$ \\
\cline { 2 - 3 } & Off & 1440 & \multirow{2}{*}{$-93 \%$} & 8091 & \multirow{2}{*}{$-53 \%$} \\
\cline { 2 - 3 } & On & 100 & & 3827 & \\
\hline
\end{tabular}

\section{B. Future scenarios: 2030 DG and 2040 GCA}

Results for 2030 DG and 2040 GCA scenarios are also provided to show scenario selection functionality other than PtG impact. Generation has been scaled for every country; it is expected VRES installed capacity to grow and some types of conventional power plants to be dismissed, and therefore their generation to be scaled down. These two scenarios represent the European efforts towards the decarbonisation.

$\mathrm{PtG}$ installed capacity and placement have not been changed from 2017 scenario results, in order to show how a solution that worked quite well in 2017 would perform if applied in future scenarios. Results are summarized in TABLE IV. for 2030 DG scenario and in TABLE V. for 2040 GCA scenario, which show how a growing VRES capacity in the network reduces the effectiveness of the deployment of the same PtG plants set. 
As it can be seen in Fig. 7 and Fig. 8, the effect of PtG units still exists, but it is way less than the one in 2017 scenario. Only the minor imbalances are absorbed, and PtG effects results in a small reduction of the biggest peaks.

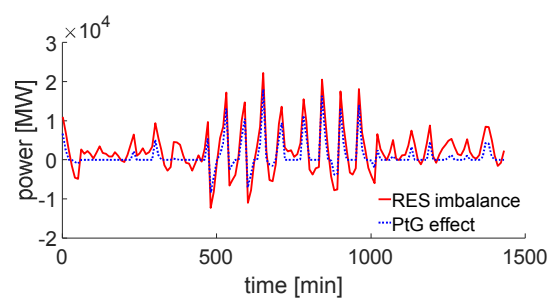

Fig. 7. Load profiles for 2030DG scenario, 1984 climatic conditions (January)

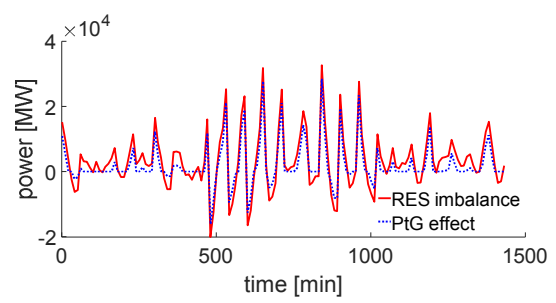

Fig. 8. Load profiles for 2040 gca scenario, 1984 climatic conditions (January)

Referring to the following summary tables, the VRES imbalance peak grows in future scenarios compared to 2017 one, given the VRES installed capacity increase. Therefore, it is necessary to plan PtG sizing and siting in a proper way, referring to future VRES penetration.

TABLE IV. PERFormanCE OF PTG IN 2017 SCENARIO

\begin{tabular}{|c|c|c|c|c|c|}
\hline \multirow[b]{2}{*}{ Scenario } & \multirow{2}{*}{$\begin{array}{c}\text { PtG } \\
\text { status }\end{array}$} & \multicolumn{4}{|c|}{ VRES imbalance } \\
\hline & & $\begin{array}{c}\text { Duration } \\
\text { [min] }\end{array}$ & $\begin{array}{c}\text { Difference } \\
{[\%]}\end{array}$ & $\begin{array}{c}\text { Peak } \\
{[M W]}\end{array}$ & $\begin{array}{c}\text { Difference } \\
{[\%]}\end{array}$ \\
\hline \multirow{2}{*}{ January } & Off & 1440 & \multirow{2}{*}{$-64 \%$} & 22186 & \multirow{2}{*}{$-19 \%$} \\
\hline & On & 520 & & 18029 & \\
\hline \multirow{2}{*}{ July } & Off & 1440 & \multirow{2}{*}{$-60 \%$} & 23895 & \multirow{2}{*}{$-18 \%$} \\
\hline & On & 580 & & 19639 & \\
\hline
\end{tabular}

TABLE V. PERformance OF PTG IN 2017 SCENARIO

\begin{tabular}{|c|c|c|c|c|c|}
\hline \multirow{3}{*}{ Scenario } & \multirow{2}{*}{$\begin{array}{c}\text { PtG } \\
\text { status }\end{array}$} & $\begin{array}{c}\text { Duration } \\
\text { [min] }\end{array}$ & $\begin{array}{c}\text { Difference } \\
\text { [\%] }\end{array}$ & $\begin{array}{c}\text { Peak } \\
{[\boldsymbol{M W}]}\end{array}$ & $\begin{array}{c}\text { Difference } \\
\text { [\%] }\end{array}$ \\
\hline \multirow{3}{*}{ January } & Off & 1440 & & 32534 & \multirow{2}{*}{ July } \\
\cline { 2 - 3 } & On & 730 & $-49 \%$ & 28477 & $-13 \%$ \\
\cline { 2 - 3 } & Off & 1440 & & 33908 & \multirow{2}{*}{$-12 \%$} \\
\cline { 2 - 3 } & On & 770 & & 29728 & \\
\hline
\end{tabular}

\section{CONCLUDING REMARKS}

The magnitude of the VRESs' impact in European power systems will increase in the next years, as it can be seen comparing 2017 scenario to the 2030 DG and 2040 GCA ones in network \#T3, almost up to three times the 2017 level, and transmission networks needs to be ready for the future. The simulation results indicate that $\mathrm{PtG}$ can effectively support the operation of the European transmission network by absorbing the VRESs' variability, which cannot be completely foreseen in advance.

The simulation results for the years 2030 and 2040 imply on the importance of generating proper future scenarios and investigating the results, despite their uncertainty. A set of PtG plants, installed in the conditions corresponding to the 2017 scenario, can reduce VRES imbalance effect duration by $\sim 92 \%$, when applied in 2030 DG and 2040 GCA scenarios sees a reduced effect (from $\sim 92 \%$ of VRES imbalance effect duration reduction to $\sim 62 \%$ and $\sim 48 \%$, respectively). The peak power reduction of the RES imbalance on the network follows a similar trend: being directly linked to the total capacity of PtG installed, the peak reduction decreases from $\sim 50 \%$ for 2017 scenario down to $\sim 18 \%$ and $\sim 12 \%$ for the future scenarios.

This has been evaluated by developing a two-step sequential code, where the first step is devoted to simulating an hourly generator dispatching based on the load values and the expected values of the VRES (whose results could be used also for other applications, such the individuation of the optimal zonal price configurations [28][29]), whereas the second step aims to re-dispatch the conventional generators according to the variations of the net load due to the difference between the expected and actual value of VRES-based power plants.

These results make room for further investigations, and the next steps for the continuation of this work will be basically two:

- the impact of optimal PtG placement on network infrastructure

- the complete analysis of the impact on the transmission system operation with long term future scenarios

These two topics are closely connected, because they are both related to the future development of the electricity grids, in terms of future investments already scheduled or under investigation.

\section{ACKNOWLEDGMENT}

This contribution has received funding from the European Union's Horizon 2020 research and innovation programme under grant agreement No. 691797 (project STORE\&GO). The paper only reflects the authors' views and the European Union is not liable for any use that may be made of the information contained herein.

The Authors would like to thank Yang Zhang and Francesco Arrigo for the fruitful discussions about the topics shown in this paper.

\section{REFERENCES}

[1] A. Mazza, E: Carpaneto, G. Chicco, and A. Ciocia, "Creation of network case studies with high penetration of distributed energy resources", Proc. - 2018 53rd Int. Universities Power Engineering Conference (UPEC 2018), Glasgow (UK, DOI:10.1109/UPEC.2018.8542025

[2] A. Ciocia, V.A. Boicea,G. Chicco, P. Di Leo, A. Mazza, E. Pons, F. Spertino, and N. Hadj-Said, "Voltage control in low-voltage grids using distributed photovoltaic converters and centralized devices", IEEE Transactions on Industry Applications, vol. 55(1), pp. 225 - 237, 2019. 
[3] H. Khani, N. El-Taweel, H. E. Z. Farag, "Power Congestion Management in Integrated Electricity and Gas Distribution Grids", IEEE Systems Journal, In press.

[4] R. Lasseter, A. Akhil, C. Marnay, J. Stephens, J. Dagle, R. Guttromson, A.S. Meliopoulous, R.Yinger, J. Eto, "Integration of distributed energy resources. The CERTS Microgrid Concept", Technical Report, 2002, https://escholarship.org/uc/item/9w88z7z1.

[5] G.Chicco, and A.Mazza, "An overview of the probability-based methods for optimal electrical distribution system reconfiguration", Proc. 4th International Symposium on Electrical and Electronics Engineering (ISEEE 2013), Galati (Romania), 11-13 Oct. 2013.

[6] W. Zappa, M. Junginger, M. van den Broek, "Is a 100\% renewable European power system feasible by 2050?", Applied Energy, vol. 233 234, pp. 1027-1050, 2019, DOI: https://doi.org/10.1016/j.apenergy.2018.08.109.

[7] A. S. Dagoumas, and N. E. Koltsaklis, "Review of models for integrating renewable energy in the generation expansion planning", Applied Energy, vol.242, pp. 1573-1587, 2019, DOI: https://doi.org/10.1016/j.apenergy.2019.03.194

[8] M. V. Loureiro, R. K. Schell, J. Claro, P. Fischbeck, "Renewable integration through transmission network expansion planning under uncertainty", Elec. Power Syst. Research, vol. 165, pp. 45-52, 2018, DOI: https://doi.org/10.1016/j.epsr.2018.07.037.

[9] E. Mortaz, and J. Valenzuela, "Evaluating the impact of renewable generation on transmission expansion planning", Elec. Power Syst. Research, vol 169, pp- 35-44, 2019, DOI: https://doi.org/10.1016/j.epsr.2018.12.007.

[10] J.Hörscha, F. Hofmann, D. Schlachtberger, and T. Brown, "PyPSAEur: An open optimisation model of the European transmission system", Energy Strategy Reviews, vol. 22, pp. 207-215, 2018, DOI: https://doi.org/10.1016/j.esr.2018.08.012.

[11] T. Fiedler, "Simulation of a power system with large renewable penetration", Renewable Energy, vol 130, pp. 319-328, 2019, DOI: https://doi.org/10.1016/j.renene.2018.06.061.

[12] J. Eickmann, T. Drees, J.D. Sprey, and A. Moser, "Optimizing Storages for Transmission System Operation", Energy procedia, vol. 46, pp. $13-$ 21, 2014, DOI: doi: 10.1016/j.egypro.2014.01.153.

[13] H. Blanco, and A. Faaij, "A review at the role of storage in energy systems with a focus on Power to Gas and long-term storage", Renewable and Sustainable Energy Reviews, vol. 81, 2018, pp. 10491086, DOI: https://doi.org/10.1016/j.rser.2017.07.062.

[14] A. Mazza, E. Bompard, and G. Chicco, "Applications of power to gas technologies in emerging electrical systems",

[15] ENTSO-E, Ten Years Network Development Plan 2016: Grid data, https://docstore.entsoe.eu/stum/, (on line 18th July 2018)
[16] B. Wiegmans, Unofficial ENTSO-E dataset processed by GridKit, https://zenodo.org/record/55853\#.WpA8cjtG2Co (on line 18th July 2018)

[17] J. Hörsch, F. Neumann and T. Brown, Python for Power System Analysis (PyPSA), Model of the European Energy System, https://github.com/PyPSA/pypsa-eur

[18] J. Hörsch, F. Hofmann, D. Schlachtberger, and T. Brown, Supplementary Data: Code, Input Data and Model data: PyPSA-Eur: An Open Optimisation Model of the European Transmission System, https://zenodo.org/record/1246852\#.W307aylaY_V (on line 19th July 2018)

[19] J. Hörsch, F. Hofmann, D. Schlachtberger, and T. Brown, PyPSA-Eur: An Open Optimisation Model of the European Transmission System, Preprint submitted to International Journal of Energy Strategy Reviews, June 2018, https://arxiv.org/pdf/1806.01613.pdf

[20] ENTSO-E, European Grid Map, https://www.entsoe.eu/data/map/ (on line on 17th July 2018)

[21] D. Oeding, B. Oswald, Elektrische Kraftwerke und Netze, 7th Edition, Springer, 2011 (in German)

[22] ENTSO-E, Power Statisics - Load, https://www.entsoe.eu/data/powerstats/hourly load/

[23] ENTSO-E, TYNDP, http://tyndp.entsoe.eu/maps-data/ (on line on 17th July 2018)

[24] ENTSO-E, TYNDP 2018 Scenario Report, https://tyndp.entsoe.eu/tyndp2018/scenario-report/

[25] ENTSO-E, Power Statisics - Generation, https://www.entsoe.eu/data/power-stats/net-gen-capacity/ (on line on 17th July 2018)

[26] ENTSO-E, Ten Years Network Development Plan 2016: Grid data, https://docstore.entsoe.eu/stum/, (on line 18th July 2018)

[27] R. D. Zimmerman, C. E. Murillo-Sanchez, and R. J. Thomas, Matpower: Steady-State Operations, Planning and Analysis Tools for Power Systems Research and Education, IEEE Transactions on Power Systems, vol. 26, pp. 12-19, Feb. 2011 http://dx.doi.org/10.1109/TPWRS.2010.2051168.

[28] G. Chicco et al., Overview of the Clustering Algorithms for the Formation of the Bidding Zones, submitted to the 54th International Universities Power Engineering Conference (UPEC 2019) Bucharest, Romania, 3-6 September 2019.

[29] C. Bovo et al., Review of the Mathematic Models to Calculate the Network Indicators to Define the Bidding Zones, submitted to the 54th International Universities Power Engineering Conference (UPEC 2019), Bucharest, Romania, 3-6 September 2019. 